\title{
Control of Poly-Articular Chain Trajectory Using Temporal Sequence of Its Joints Displacements
}

\author{
Pierre Legreneur ${ }^{1,2}$, Thomas Creveaux ${ }^{2}$, Vincent Bels ${ }^{1}$ \\ ${ }^{1}$ Departement Ecologie et Gestion de la Biodiversité, Muséum National d'Histoire Naturelle, Paris Cedex, France \\ ${ }^{2}$ Université de Lyon, Villeurbanne Cedex, France \\ E-mail: pierre.legreneur@univ-lyon1.fr, thomas.creveaux@gmail.com,bels@mnhn.fr \\ Received November 9, 2010; revised December 9, 2010; accepted December 20, 2010
}

\begin{abstract}
This paper discusses on the role of joint temporal sequence while moving a two-dimensional arm from an initial position to targets into the fingertip workspace in humans. For this purpose, we proposed a general monotonic model of joint asymmetric displacement. Optimization consisted in minimizing least square displacement of either fingertip or arm centre of mass from arm initial position to four targets located into fingertip workspace, i.e. contralaterally and ipsilaterally. Except for $60^{\circ}$ ipsilateral target, results of the simulation presented in all cases temporal sequences of the shoulder, the elbow and the wrist. We concluded that primary function of proximal-to-distal or distal-to-proximal joint sequence is to flatten the trajectory of the fingertip or body centre of mass.
\end{abstract}

Keywords: Coordination, Modeling, Simulation, Sigmoid, Pointing, Jumping

\section{Introduction}

Explosive movements like leaping or jumping are characterized by production of maximal external force at the interface between the substrate and the body during a short time. During such movements, most results presented in the literature exhibits a proximal-to-distal sequence of the main engaged limbs, i.e. the most proximal joint peak velocity occurs before the distal ones. These coordinations have been reported for various human movements, e.g. squat jumping [1], sprint push-off [2], handball throwing [3], football kick while running and walking [4,5], speed skating [6] or artistic ice-skating [7]. This sequence of coordination also characterize many tetrapod taxa using jumping in terrestrial and arboreal habitats, i.e. bonoboo [8], Galago [9,10], Rana esculenta [11]. Thus, this sequence appears in various species with highly different morphologies, occupying different ecological niches and interacting into various trophic striates. Consequently, it may reflect mechanical constraints of the musculo-skeletal systems engaged in animal and human locomotions [12]. The more the joint approaches its maximal extension, the less the transformation of the segment angular velocity into linear velocity is effective [13]. It is necessary to reduce the velocity of the joint before its maximal extension (anatomical constraint) in order to protect this joint from any damage. The proximal-to-distal sequence allows to delay the negative influence of the anatomical and geometrical constraints in explosive movements [14]. This sequence contributes to produce, transfer and orientate mechanical power, from proximal to distal segments through mono- and bi-articular musculo-tendon systems crossing the limb joints [15-17].

Although proximal-to-distal sequence is well documented in explosive movements, less information is available in the literature on poly-articular coordinations in slow movements, i.e. pointing or grasping tasks [18]. However, by about 24 months of age in humans, proximal-to-distal sequence emerge in vertical pointing task between shoulder flexion and elbow extension [19]. Unpublished results obtained in pointing task experiments conducted in our lab showed that proximal-to-distal sequence was mainly chose by subjects whatever the position of the target in their arm workspace, i.e. contralateral (75\%) vs. ipsilateral (75\%) vs. frontal (50\%), proximal (60\%) vs. distal (80\%). From these results reported as well in explosive (jumping) as velocity unconstraint (pointing) movements, we conclude that proximal-todistal coordination appears whatever the spatio-temporal typology of the movement, and could not be only interpreted under power transfer mechanisms. 
As shown in Figure 1, a common characteristic of all previously described movements is the linear or quasilinear shape of the body centre of mass (CoM) of the subjects or the distal extremity of the moving poly-articular chain, e.g. the fingertip in pointing task. Fingertip trajectory concavity depends also on the spatial position of the target to reach and increase from ipsilateral to contralateral sides [20]. In human jumping and animal leaping, we showed that, whereas the magnitude of the velocity vector increased all along the CoM trajectory during push-off, its orientation was reached at $20 \%$ of total push-off time in Microcebus murinus [21] and at $40 \%$ in humans [22] and then remained constant. In other words, after an orientation phase of the body CoM, these species tend to maintain their trajectory linear. Trajectories of fingertip or CoM result from spatio-temporal organizations of limb segments, i.e. shoulder, elbow and wrist in pointing task and hip, knee, ankle and metatarso-phalangeal joint in jumping. If we suppose that these joints move through rotations in a strictly monotonic manner, i.e. exclusive flexion or extension, it is obvious that simultaneous displacements of joints composing a poly-articular chain will induce angular displacement of its distal extremity or CoM. Thus, we can predict that linear trajectory of distal end or CoM of a poly-articular chain results necessary from temporal joints sequence. The main purpose of the present theoretical study was then to test this prediction.

\section{General Model of Joint Displacement}

Throughout this paper, we state that the joint angle displacement is a monotonic function of time and that no regulation of the trajectory by the central nervous system occurs during the whole joint displacement. Consequently, joint displacement was modelled through a sigmoid profile (Figure 2(a)) and a velocity bell-shaped shape (Figure 2(b)) [23,24]. These shapes account for synergistic actuators activations at a joint, i.e. agonist and antagonist musculo-tendon systems.

At the start of the movement, the kinematics of the joint is given by

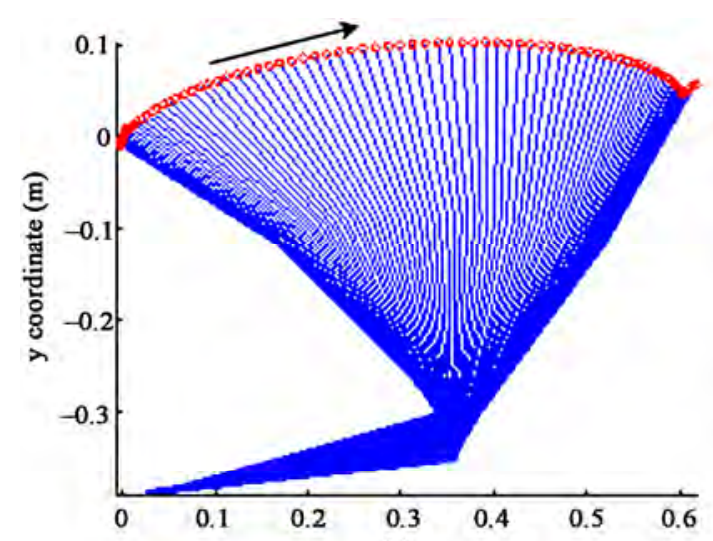

(a)

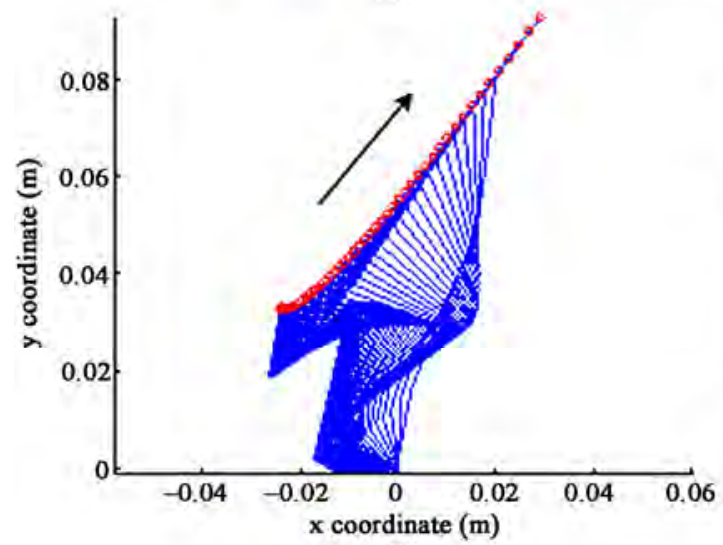

(c)

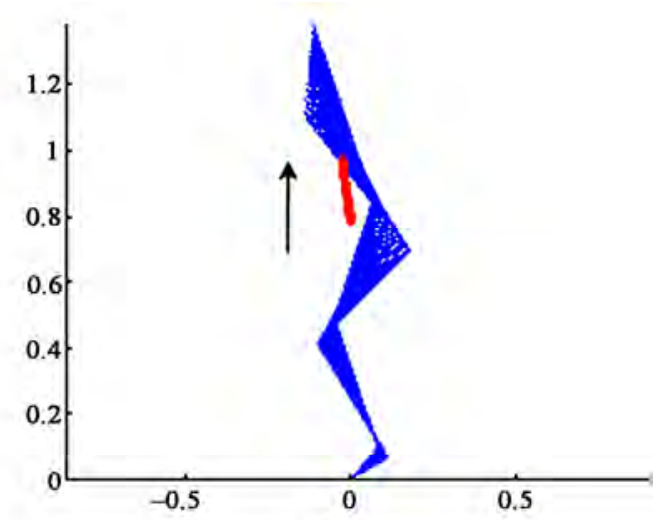

(b)

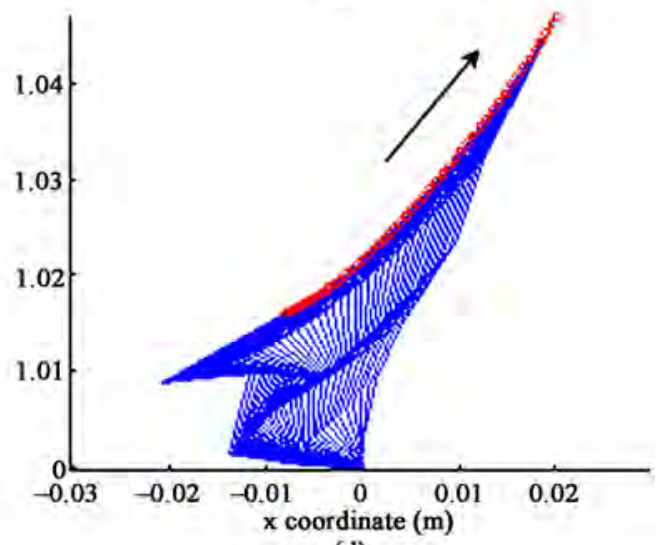

(d)

Figure 1. Stick diagrams of pointing or jumping in human and animals. Arrows indicate the sense of the movements. (a) Arm pointing task in human to a ipsilateral and distal target. Red circles represent the fingertip; (b) Push-off phase in human squat jumping. Red circles represent the body centre of mass; (c) and (d) Push-off phase in maximal high leaping in Microcebus murinus and Anolis carolinensis. Red circles represent their body mass centres assimilated to their iliac crest. 


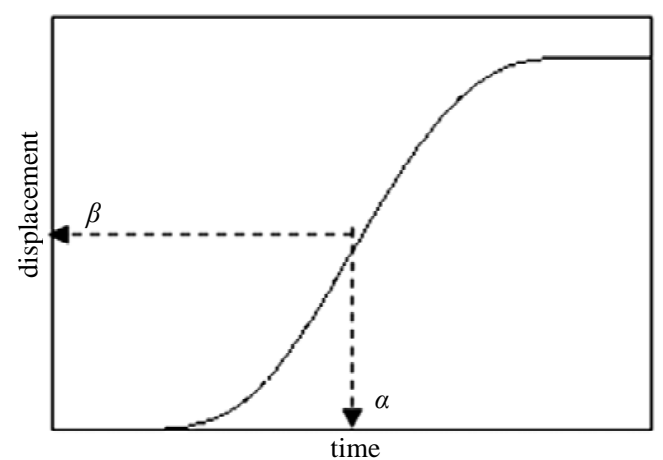

(a)

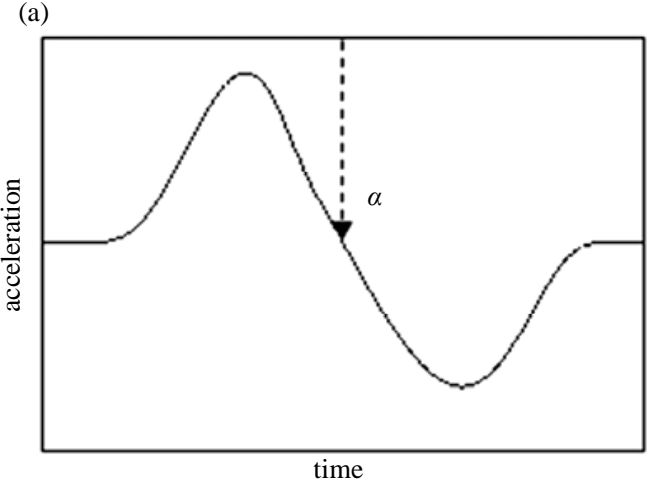

(c)

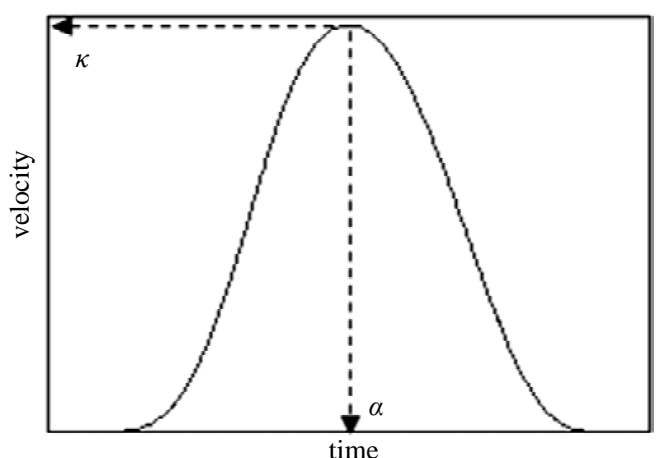

(b)

Figure 2. Normalized time histories of general joint kinematics. (a) Normalized angular displacement: angle $\beta$ is achieved at instant $\alpha$; (b) Normalized angular velocity: peak velocity $\kappa$ is achieved at instant $\alpha$; (c) Normalized angular acceleration allowed characterizing a biphasic joint displacement: instant $\alpha$ corresponds to zero acceleration.

$$
\left\{\begin{array}{l}
\theta\left(t_{i}\right)=\theta_{i} \\
\dot{\theta}\left(t_{i}\right)=0 \forall t \leq t_{i} \\
\ddot{\theta}\left(t_{i}\right)=0
\end{array}\right.
$$

Similarly, at the end of the joint rotation,

$$
\left\{\begin{array}{l}
\theta\left(t_{f}\right)=\theta_{f} \\
\dot{\theta}\left(t_{f}\right)=0 \forall t \geq t_{f} \\
\ddot{\theta}\left(t_{f}\right)=0
\end{array}\right.
$$

Thus, the general formulation of joint displacement is given by

$$
(t)\left\{\begin{array}{lc}
\theta_{i} & \forall t<t_{i} \\
\theta_{f} & \forall t>t_{f} \\
\theta_{i}+\left(\theta_{f}-\theta_{i}\right) \sigma(\tau) \text { with } \tau=\left(t-t_{i}\right) /\left(t_{f}-t_{i}\right) \\
& \forall t \in\left[t_{i} ; t_{f}\right]
\end{array}\right.
$$

where $\sigma$ is a function defined on $[0,1]$ such as $\sigma(0)=\sigma(1)-1=0$. This function is at least of class $C^{2}$, i.e. is at least twice continuous and differentiable. Moreover, $\sigma$ has to account for the asymmetric profile of joint angular velocity reported experimentally [25]. It should be noted that this asymmetry should be modified by several constraints. Thus, it increases with spatial accuracy demands [26]. Moreover, the faster is the movement, the longer is the acceleration phase [27]. In other words, the asymmetry of the velocity peak should be inverted if the velocity exceeds a specific threshold. Many equations are available in the literature to model symmetric sigmoid shapes. Fewer equations were developed for asymmetric shapes, i.e. plant growth [28] or handwriting [29]. However, they are of class $C^{1}$ or do not satisfy equation (3). So, we developed an original sigmoid model $\sigma$ of class $C^{\infty}$ as:

$$
\begin{gathered}
\sigma(\tau)=\frac{\int_{0}^{\tau} h_{\lambda, \mu, a}(y) d y}{\int_{0}^{1} h_{\lambda, \mu, a}(y) d y} \\
h_{\lambda, \mu, a}(y)= \begin{cases}f_{\lambda, \mu}(y) g_{a, \alpha}(y) & \forall a>0, y \in[0, \alpha[ \\
f_{\lambda, \mu}(y) & \forall a>0, y \in[\alpha, 1] \\
f_{\lambda, \mu}(y) & \forall a<0, y \in[0, \alpha] \\
f_{\lambda, \mu}(1-y) g_{-a, 1-\alpha}(1-y) & \forall a<0, y \in] \alpha, 1]\end{cases}
\end{gathered}
$$


where $\alpha$ represents the normalized time at which normalized angular peak velocity $\kappa$ is attained. This instant corresponds to normalized joint position $\beta$. These are given by

$$
\begin{aligned}
& \alpha=\frac{t_{0}-t_{i}}{t_{f}-t_{i}} \\
& \beta=\frac{\theta_{0}-\theta_{i}}{\theta_{f}-\theta_{i}} \\
& \kappa=\frac{K\left(t_{f}-t_{i}\right)}{\theta_{f}-\theta_{i}}
\end{aligned}
$$

where $K$ represents absolute angular peak velocity. Thus, these parameters are linked through the following relations:

$$
\left\{\begin{array}{l}
\sigma(\alpha)=\beta \\
\dot{\sigma}(\alpha)=\kappa \\
\ddot{\sigma}(\alpha)=0
\end{array}\right.
$$

$f_{\lambda, \mu}$ and $g_{a, \alpha}$ are given by

$$
\left\{\begin{array}{l}
f_{\lambda, \mu}^{(n)}(0)=0 \\
\forall y \in] 0,1\left[, \quad f_{\lambda, \mu}(y)=e^{-\left(\frac{1}{y(1-y)^{\mu}}\right)^{\lambda}}\right. \\
f_{\lambda, \mu}^{(n)}(1)=0
\end{array}\right.
$$

And

$$
g_{a, \alpha}(y)= \begin{cases}1 & \forall a=0 \\ 1-e^{\frac{t}{a(t-\alpha)}} & \forall a>0, y \in[0, \alpha[ \\ 1 & \forall a>0, y \in[\alpha, 1]\end{cases}
$$

Solving Equation (9) allows posing

$$
\alpha=\frac{1}{1+\mu}
$$

From Equations (9) and (11), we can deduce $\kappa=$ $f_{\lambda, \mu}(\alpha)$ :

$$
\kappa=e^{-\left[(1+\mu)\left(\frac{\mu}{1+\mu}\right)^{-\mu}\right]^{\lambda}}
$$

Typical time courses of both joint displacement and angular velocity are given in Figure 3 for various $\alpha$ values. That illustrates the flexibility of the model for simulating asymmetric shaped curves on both left $(\alpha<0.5)$ and right $(\alpha>0.5)$ sides.

\section{Poly-Articular Chain Model}

Considering a poly-articular chain composed of a set of $p$ points $A_{p}$ such as $A_{0}$ is the origin of the reference frame $\mathfrak{R}\left(A_{0}, \vec{i}, \vec{j}\right)$, angular positions of the articulations are given by
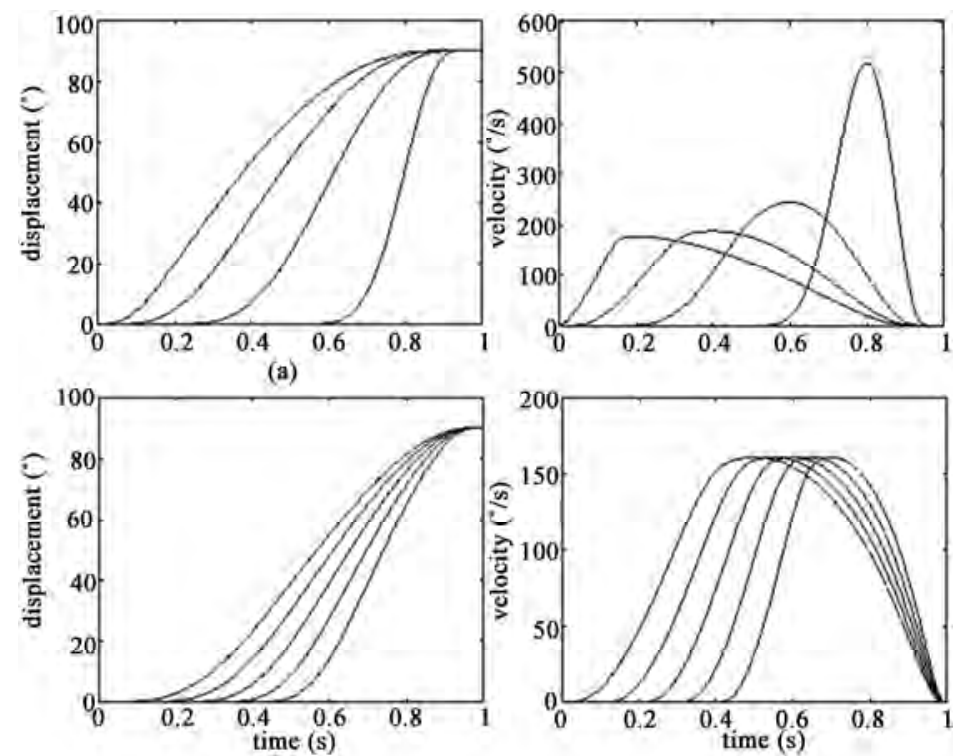

(b)

Figure 3. Absolute time histories of general joint displacement for various parameterizations. (a) Angular displacement and velocity profiles with instant of velocity peak $\alpha \in\{0.2,0.4,0.6,0.8\} \cdot t_{i}, t_{f}, \theta_{i}, \theta_{f}, a$ and $\lambda$ were fixed at $0,1,0,90,1$ and $\alpha$ respectively; (b) Angular displacement and velocity profiles with beginning of the movement $t_{i} \in\{0.1,0.2,0.3,0.4\}$. $t_{f}, \theta_{i}, \theta_{f}, \alpha, a$ and $\lambda$ were fixed at $1,0,90,0.5,1$ and 0.5 respectively. 


$$
\left\{\begin{array}{l}
\theta_{1}=\left(\vec{\imath}, \overrightarrow{\overrightarrow{\mathrm{A}_{0} \mathrm{~A}_{1}}}\right) \\
\forall i \in\{2, \cdots, p\}, \theta_{i}=\left(\overrightarrow{A_{l-2} A_{l-1}}, \overrightarrow{A_{t-1} A_{\imath}}\right)
\end{array}\right.
$$

with the constraint

$$
\forall i \in\{1, \cdots, p\}, \theta_{i} \in\left[\theta_{i}^{-}, \theta_{i}^{+}\right]
$$

Segment lengths $l_{i}$ are defined as

$$
\forall i \in\{1, \cdots, p\}, \quad l_{i}=A_{i-1} A_{i}
$$

Thus, the coordinates of $A_{j}$ in the reference frame $\mathfrak{R}$ are deduced from

$$
\overrightarrow{A_{0} A_{j}}\left\{\begin{array}{l}
x_{j}=\sum_{i=1}^{j} l_{i} \cos \left(\sum_{k=1}^{i} \theta_{i}\right) \\
y_{j}=\sum_{i=1}^{j} l_{i} \sin \left(\sum_{k=1}^{i} \theta_{i}\right)
\end{array}\right.
$$

To model a human upper limb, we considered one subject of $1.80 \mathrm{~m}$ height. Lengths and positions of CoM of the segments constituting the upper limb were determined from anthropometric tables [30]. Lengths are presented as percent of total body height $(0.108,0.146$ and 0.186 for the hand, forearm and upper arm respectively) as well as distance between proximal end of segments and their CoM (0.506, 0.430 and 0.436 for the hand, forearm and upper arm respectively) and their masses (0.006, 0.016 and 0.028 for the hand, forearm and upper arm respectively). One degree of freedom was considered per arm joint, i.e. shoulder abduction/adduction, elbow flexion/extension and wrist abduction/adduction. The angles corresponding to minima and maxima of joints displacement are respectively $-60^{\circ} / 120^{\circ}$ for the shoulder $\left(\theta_{S}\right), 0^{\circ} / 130^{\circ}$ for the elbow $\left(\theta_{E}\right)$ and $-10^{\circ} / 25^{\circ}$ for the wrist $\left(\theta_{W}\right)$ (Figure $4(\mathbf{a})$ ). $0^{\circ}$ corresponds to joint extension. These degrees of freedom of the arm allow defining the workspace of the fingertip in a two-dimensional space (Figure 4(b)) [31].

For all targets, initial arm position was set so that the fingertip was located in front of the shoulder at a distance of $30 \%$ of the arm length. The target distance was set at $80 \%$ of arm length for all targets. The spatial angles of the four distal targets were set at $20^{\circ}$ and $60^{\circ}$ ipsilaterally, and $120^{\circ}$ and $160^{\circ}$ contralaterally (Figure 4(b)). At the beginning of the movement, angular positions of the shoulder, the elbow and the wrist were determined using classical inverse geometrical procedure $\left(\theta_{S}=0^{\circ}\right.$, $\theta_{E}=130^{\circ}, \theta_{S}=25^{\circ}$ ).

\section{Upper Limb Joints Coordinations in Simulated Pointing Task}

For each joint of the arm, three control parameters were considered, i.e. $\lambda, \mu, a$ and $\theta_{f}$. Optimization consisted in minimizing least square displacement of either fingertip or arm CoM. Each position was calculated over $1 \mathrm{~s}$ movement at a frequency of $100 \mathrm{~Hz}$. Consequently, performance criterion $J$ is given by:

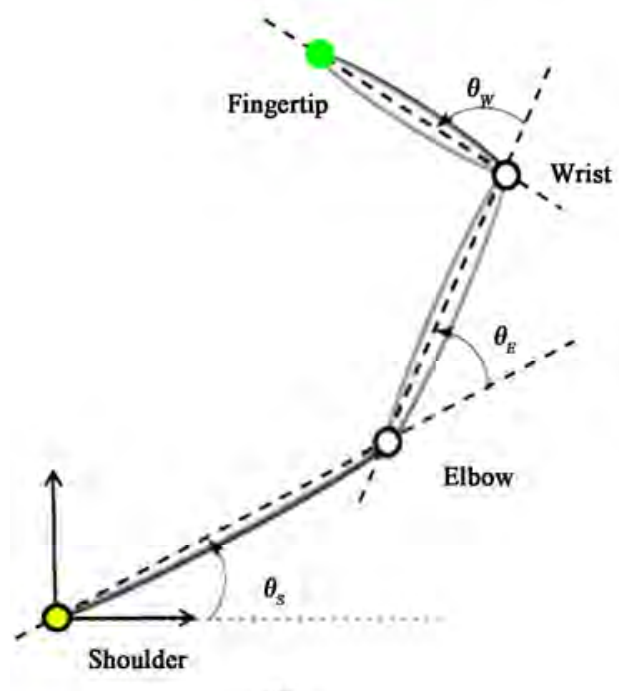

(a)

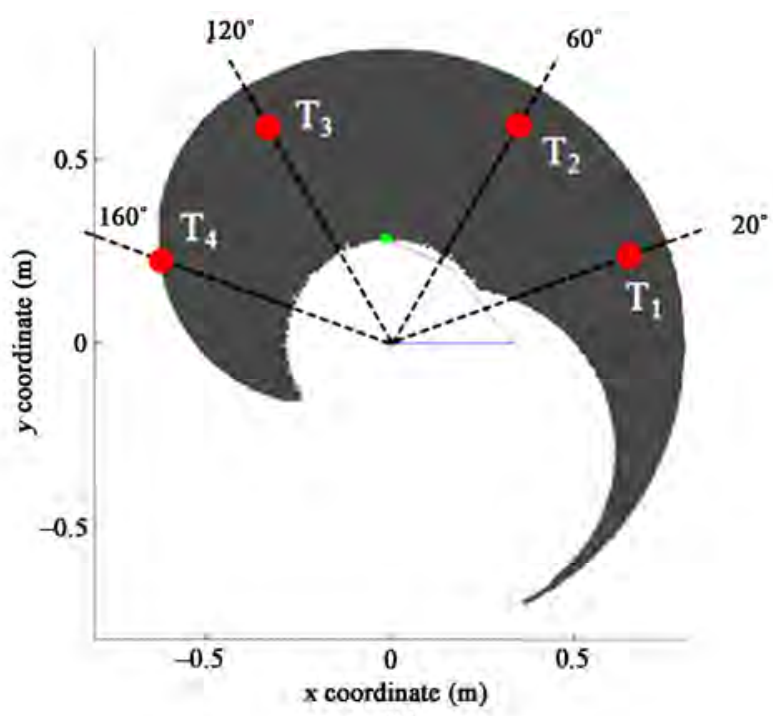

(b)

Figure 4. (a) Planar schematic representation of the arm and its reference frame. $\theta_{S}, \theta_{E}$ and $\theta_{W}$ represent the shoulder, elbow and wrist joint positions. Maximal extension corresponds to $0^{\circ}$; (b) Initial and final positions of the arm into the workspace of the fingertip (area traced in gray). Four targets are considered $\left(T_{1}\right.$ to $\left.T_{4}\right) . T_{1}$ and $T_{2}$ are located ipsilaterally. $T_{3}$ and $\mathbf{T}_{4}$ are located contralaterally. 


$$
J=\sum_{i=2}^{101} \sqrt{\left(x_{i}-x_{i-1}\right)^{2}+\left(y_{i}-y_{i-1}\right)^{2}}
$$

The fingertip trajectory was constrained by the minimal distance between its position at the end of the pointing task and the target. Moreover, the fingertip final position was constrained to be at most $5 \mathrm{~mm}$ far from the target. Optimization procedure was performed under Matlab ${ }^{\circledR}$ 7.3.0 software (Mathworks Inc., Natick, MA, USA).

Results of the simulation are presented in Table $\mathbf{1}$, and kinematics of the arm is showed in Figure 5 for $20^{\circ}$ ipsilateral target when either fingertip or arm CoM trajectories are optimized. The use of continuous and monotonic functions for joints' displacements induced that the fingertip is unable to follow a straight line (Figure 5a). Indeed, the hand path presented a curvature, as usually observed in the literature [20]. The purpose of the optimization procedure was to flatten fingertip or arm CoM trajectories. Considering instant of peak velocity $\alpha$, for both optimizations (fingertip vs arm CoM) and all targets, joints moved simultaneously only for $60^{\circ}$ ipsilateral target and arm CoM optimization. In all others cases, temporal sequences are predicted. Regarding to shoulder and elbow joints, these sequences are either proximal-todistal into the ipsilateral space and distal-to-proximal into the contralateral one. Concerning the wrist, the se- quence was inconsistent, i.e. the wrist moved before the elbow or after.

\section{Conclusions}

The trajectory shape of a point of a poly-articular chain, or its CoM depends on the temporal organisation of its constitutive joints displacements. Moreover, we demonstrated that minimization of path length, or linearization of this trajectory imposes a temporal sequence of its joints displacements if these are continuous and monotonic. This sequence is either proximal-to-distal or distal-to-proximal in function of the location of the ending position into the workspace. In dynamic locomotion, like jumping, coordinations are always proximal-to-distal. That supports the idea that the primary function of proximal-to-distal sequence in dynamic movement is to flatten the animal CoM. The secondary function of this sequence will be to transfer muscle power produce by mono-articular muscles to the ground through bi-articular muscles [1] as well as orientate reaction force [16]. Moreover, like model where the magnitude of the jerk was minimized [24], this theoretical analysis, based only on the kinematics of the movement, without any optimization of the musculo-tendon systems activation, is able to successfully reproduce hand motion.

Table 1. Control parameters values of arm joint angle kinematics for each ending position of the fingertip. For each position, values are given for digit and arm centre of mass (CoM) trajectory optimizations.

\begin{tabular}{|c|c|c|c|c|c|c|c|c|c|}
\hline & & \multicolumn{2}{|c|}{$20^{\circ}$} & \multicolumn{2}{|c|}{$60^{\circ}$} & \multicolumn{2}{|c|}{$120^{\circ}$} & \multicolumn{2}{|c|}{$160^{\circ}$} \\
\hline & & Digit & CoM & Digit & CoM & Digit & CoM & Digit & CoM \\
\hline \multirow{4}{*}{ Shoulder } & $\lambda$ & 1.50 & 0.99 & 1.32 & 1.05 & 1.31 & 0.60 & 0.85 & 0.63 \\
\hline & $\mu$ & 1.50 & 1.25 & 0.80 & 0.96 & 1.32 & 1.39 & 1.41 & 1.46 \\
\hline & $a$ & -1.11 & -1.02 & -1.00 & -1.00 & -0.20 & -1.02 & -0.65 & -1.06 \\
\hline & $\alpha$ & 0.40 & 0.45 & 0.56 & 0.51 & 0.43 & 0.42 & 0.42 & 0.41 \\
\hline \multirow{4}{*}{ Elbow } & $\theta_{f}\left({ }^{\circ}\right)$ & 74 & 78 & 75 & 81 & 81 & 79 & 76 & 78 \\
\hline & $\lambda$ & 1.50 & 1.05 & 0.72 & 0.95 & 1.39 & 1.47 & 1.33 & 1.50 \\
\hline & $\mu$ & 0.64 & 0.76 & 0.67 & 1.04 & 1.26 & 0.68 & 1.15 & 0.63 \\
\hline & $\alpha$ & 0.60 & 0.57 & 0.44 & 0.49 & 0.47 & 0.60 & 0.52 & 0.61 \\
\hline \multirow{5}{*}{ Wrist } & $\theta_{f}\left(^{\circ}\right)$ & 7 & -10 & 5 & -10 & -9 & -5 & 1 & -2 \\
\hline & $\lambda$ & 1.50 & 1.00 & 0.96 & 1.00 & 1.50 & 1.03 & 1.08 & 1.09 \\
\hline & $\mu$ & 0.38 & 0.99 & 0.95 & 1.00 & 1.12 & 0.95 & 0.96 & 0.88 \\
\hline & $a$ & -0.88 & -1.00 & -1.00 & -1.00 & -1.35 & -1.00 & -1.04 & -0.99 \\
\hline & $\alpha$ & 0.73 & 0.50 & 0.51 & 0.50 & 0.47 & 0.51 & 0.51 & 0.53 \\
\hline
\end{tabular}




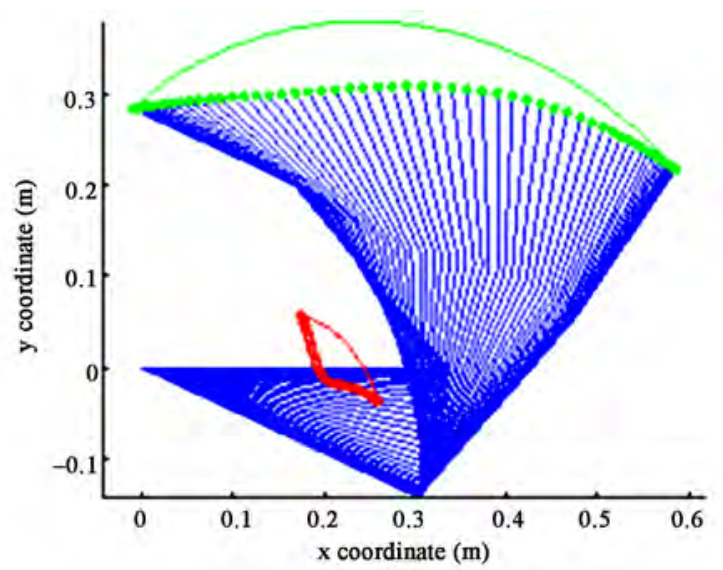

(a)
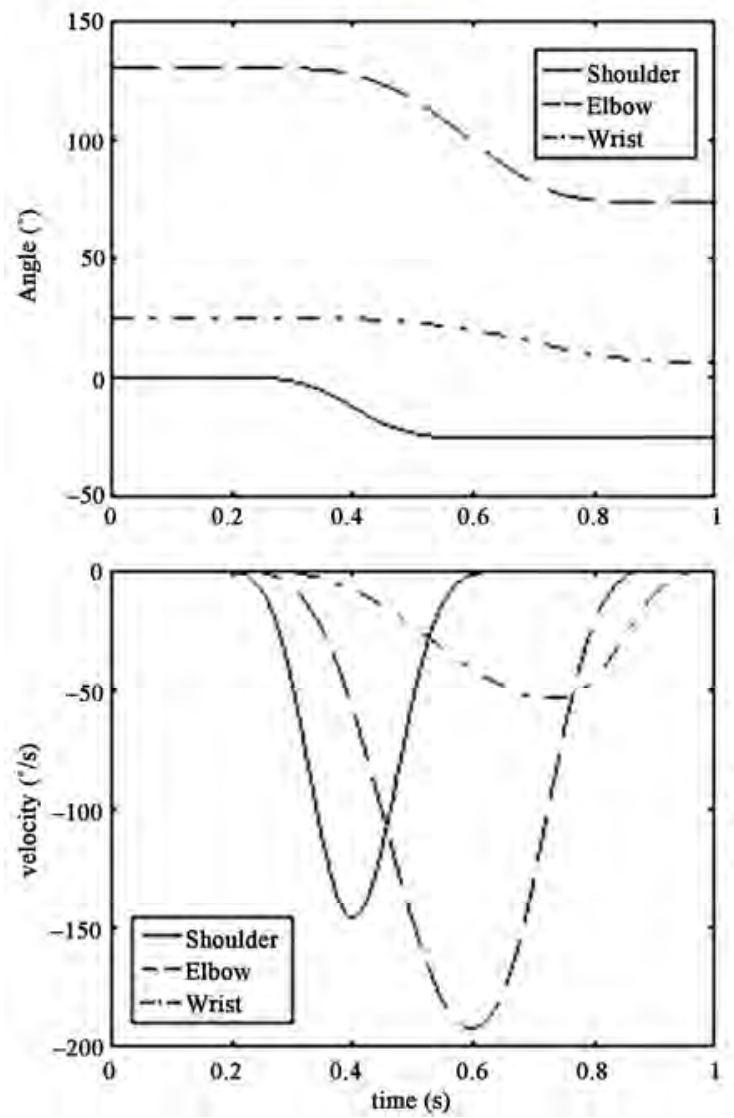

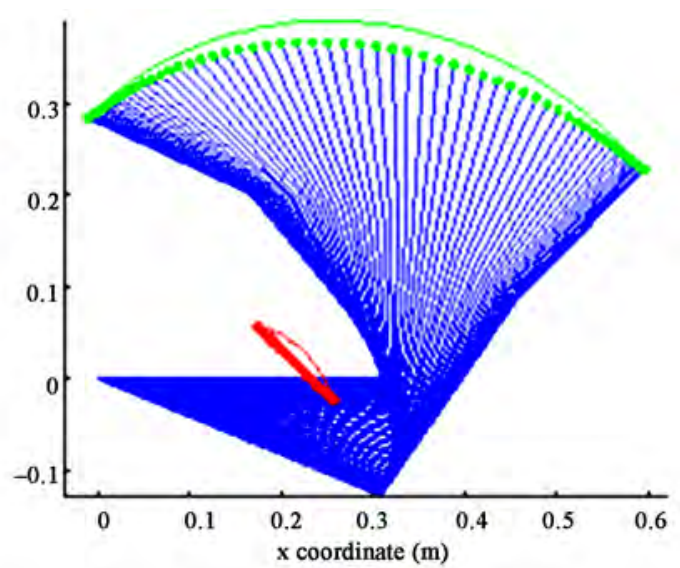

(b)
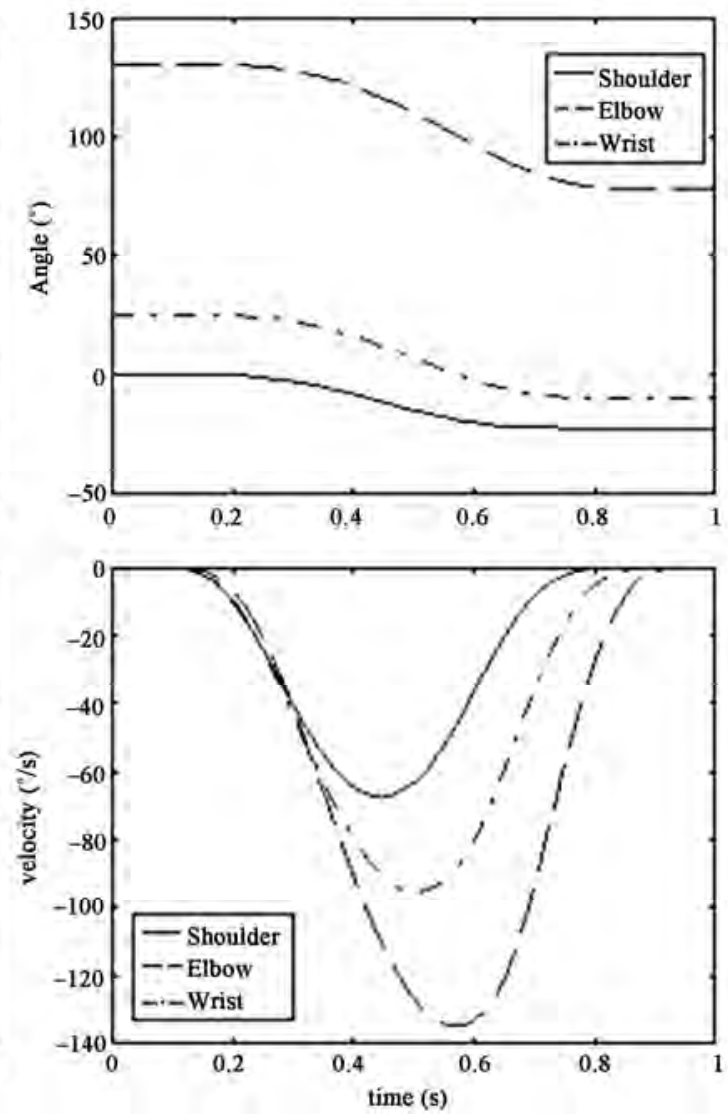

Figure 5. Predicted kinematics of pointing task for $20^{\circ}$ ipsilateral target. (a) and (b) correspond to fingertip and arm centre of mass (CoM) trajectory optimizations respectively. Stick diagrams of the arm motion are compared with fingertip and CoM trajectories in case of simultaneous displacements of the joints (thin lines). Below are represented time histories of shoulder, elbow and wrist angular displacements and velocities.

\section{Acknowledgements}

This project was conducted under the program ANR06BLAN-132-02 and the ATM program of the Museum National d'Histoire Naturelle (Paris, France) entitled "Formes possibles, formes réalisées". We would like to thank Jérôme Bastien for helping us to model joint dis- placement.

\section{References}

[1] L. Gregoire, H. E. Veeger, P. A. Huijing and G. J. van I. Schenau, "Role of Mono- and Biarticular Muscles in Explosive Movements,” International Journal of Sports Me- 
dicine, Vol. 5, No. 6, 1984, pp. 301-305. doi:10.1055/s2008-1025921

[2] R. Jacobs and G. J. van I. Schenau, "Intermuscular Coordination in a Sprint Push-off," Journal of Biomechanics, Vol. 25, No. 9, 1992, pp. 953-965. doi:10.1016/0021-92 90(92)90031-U

[3] H. Jöris, A. Muyen, G. J. van I. Schenau and H. Kemper, "Force, Velocity and Energy Flow during the Overarm Throw in Female Handball Players," Journal of Biomechanics, Vol. 18, No. 6, 1985, pp. 409-414. doi:10.1016/ 0021-9290(85)90275-1

[4] C. Putnam, "A Segment Interaction Analysis of Proximalto-Distal Sequential Segment Motion Patterns,” Medicine and Science in Sports and Exercise, Vol. 23, No. 1, 1991, pp. 130-144. doi:10.1249/00005768-199101000-00019

[5] J. Van Der Kamp, “A Field Simulation Study of the Effectiveness of Penalty Kick Strategies in Soccer: Late Alterations of Kick Direction Increase Errors and Reduce Accuracy,” Journal of Sports Sciences, Vol. 24, No. 5, 2006, pp. 467-477. doi:10.1080/02640410500190841

[6] G. J. van I. Schenau and K. Bakker, "A Biomechanical Model of Speed Skating," Journal of Human Movement Studies, Vol. 6, 1980, pp. 1-18.

[7] M. Haguenauer, P. Legreneur and K. M. Monteil, "Influence of Figure Skating Skates on Vertical Jumping Performance,” Journal of Biomechanics, Vol. 39, No. 4, 2006, pp. 699-707. doi:10.1016/j.jbiomech.2005.01.005

[8] M. N. Scholz, K. D’Aout, M. F. Bobbert and P. Aerts, "Vertical Jumping Performance of Bonobo (Pan Paniscus) Suggests Superior Muscle Properties," Proceedings of Biological Sciences, Vol. 273, No. 1598, 2006, pp. 21772184. doi:10.1098/rspb.2006.3568

[9] P. Aerts, "Vertical Jumping in Galago Senegalensis: The Quest for an Obligate Mechanical Power Amplifer,” Philosophical Transactions of the Royal Society London B, Vol. 353, 1998, pp. 1607-1620. doi:10.1098/rstb.1998.03 13

[10] M. M. Gunther, "Biomechanische Voraussetzungen beim Absprung des Senegalgalagos,” Zeitschrift für Morphologie und Anthropologie, Vol. 75, No. 3, 1985, pp.287-306.

[11] S. Nauwelaerts and P. Aerts, "Propulsive Impulse as a Covarying Performance Measure in the Comparison of the Kinematics of Swimming and Jumping in Frogs," Journal of Experimental Biology, Vol. 206, No. 23, 2003, pp. 4341-4351.

[12] G. J. van I. Schenau, "From Rotation to Translation: Constraints on Multi-joint Movements and the Unique Action of Bi-articular Muscles," Human Movement Science, Vol. 8, 1989, pp. 301-337. doi:10.1016/0167-9457 (89)90037-7

[13] R. M. Alexander, "Mechanics of Posture and Gait of Some Large Dinosaurs," Zoological Journal of the Linnean Society, Vol. 83, 1985, pp. 1-25. doi:10.1111/j.109 6-3642.1985.tb00871.x

[14] M. F. Bobbert and G. J. van I. Schenau, "Coordination in Vertical Jumping,” Journal of Biomechanics, Vol. 21, No. 3, 1988, pp. 249-262. doi:10.1016/0021-9290(88)90175-3
[15] G. J. van I. Schenau, M. F. Bobbert and R. H. Rozendal, "The Unique Action of Bi-articular Muscles in Complex Movements,” Journal of Anatomy, Vol. 155, 1987, pp. 1-5.

[16] R. Jacobs and G. J. van I. Schenau, "Control of an External Force in Leg Extensions in Humans,” Journal of Physiology, Vol. 457, 1992, pp. 611-626.

[17] R. Jacobs, M. F. Bobbert and G. J. van I. Schenau, "Mechanical Output from Individual Muscles during Explosive Leg Extensions: The Role of Biarticular Muscles," Journal of Biomechanics, Vol. 29, No. 4, 1996, pp. 513523. doi:10.1016/0021-9290(95)00067-4

[18] F. Lacquaniti and J. F. Soechting, "Coordination of Arm and Wrist Motion during a Reaching Task," Journal of Neuroscience, Vol. 2, No. 4, 1982, pp. 399-408.

[19] J. Konczak, and J. Dichgans, "The Development toward Stereotypic Arm Kinematics during Reaching in the First 3 Years of Life,” Experimental Brain Research, Vol. 117, No. 2, 1997, pp. 346-354. doi:10.1007/s002210050228

[20] Y. W. Tseng and J. P. Scholz, "The Effect of Workspace on the Use of Motor Abundance," Motor Control, Vol. 9, No. 1, 2005, pp. 75-100.

[21] P. Legreneur, F. R. Thévenet, K. M. Monteil, S. J. Montuelle, E. Pouydebat and V. Bels, "Hindlimb Interarticular Coordinations in Microcebus Murinus in Maximal Leaping,” Journal of Experimental Biology, Vol. 213, No. 8, 2010, pp. 1320-1327. doi:10.1242/jeb.041079

[22] M. Haguenauer, P. Legreneur, and K. M. Monteil, "Vertical Jumping Reorganization with Aging: A Kinematic Comparison between Young and Elderly Men,” Journal of Applied Biomechanics, Vol. 21, No. 3, 2005, pp. 236246.

[23] J. F. Soechting and F. Lacquaniti, "Invariant Characteristics of a Pointing Movement in Man," Journal of Neuroscience, Vol. 1, No. 7, 1981, pp. 710-720.

[24] T. Flash and N. Hogan, "The Coordination of Arm Movements: An Experimentally Confirmed Mathematical Model," Journal of Neuroscience, Vol. 5, No. 7, 1985, pp. 16881703.

[25] H. N. Zelaznik, R. A. Schmidt and S. Gielen, "Kinematic Properties of Rapid Aimed Hand Movements," Journal of Motor Behavior, Vol. 18, No. 4, 1986, pp. 353-372.

[26] T. E. Milner and M. M. Ijaz, "The Effect of Accuracy Constraints on Three-Dimensional Movement Kinematics,” Neuroscience, Vol. 35, No. 2, 1990, pp. 365-374. doi:10.1016/0306-4522(90)90090-Q

[27] R. Plamondon, "A Kinematic Theory of Rapid Human Movements,” Biological Cybernetics, Vol. 72, No. 4, 1995, pp. 309-320. doi:10.1007/BF00202786

[28] X. Yin, J. Goudriaan, E. A. Lantinga, J. Vos and H. J. Spiertz, "A Flexible Sigmoid Function of Determinate Growth,” Annals of Botany (London), Vol. 91, No. 3, 2003, pp. 361-371. doi:10.1093/aob/mcg029

[29] R. Plamondon and M. Djioua, "A Multi-level Representation Paradigm for Handwriting Stroke Generation," Human Movement Science, Vol. 25, No. 4-5, 2006, pp. 586-607. doi:10.1016/j.humov.2006.07.004 
[30] D. A. Winter, "Biomechanics and Motor Control of Human Movement," 4th Edition, John Wiley \& Sons, Inc., Hoboken, 2009. doi:10.1002/9780470549148

[31] J. Bastien, P. Legreneur and K. Monteil, “A Geometrical
Alternative to Jacobian Rank Deficiency Method for Planar Workspace Characterisation," Mechanism and Machine Theory, Vol. 45, No. 2, 2010, pp. 335-348. doi:10. 1016/j.mechmachtheory.2009.09.007 